\title{
Research of Structural Reliability Analysis Based on Nonlinear Weighted Response Surface Methodology
}

\author{
Mingren $\mathrm{Xu}^{1}$, a Zongjie $\mathrm{CaO}^{2, \mathrm{~b}}$ \\ ${ }^{1}$ Naval Aeronautical University. Department of Airborne Vehicle Engineering, Yantai 264001,China; \\ ${ }^{2}$ Aviation University of Air Force. Department of Aviation Mechanical Engineering, Changchun \\ 130022, China \\ a xumingren2016@163.com, b caozongjie2000@163.com
}

\section{Keywords: Response Surface Methods, Structural Reliability, Weighted regression}

\begin{abstract}
Response surface method(RSM) is one of the effective methods to solve the reliability problem of implicit structure. In this paper, a new weighting method is proposed based on the response surface method to solve the nonlinear problem. The weighted design method is used to determine the design point of the response surface, a certain number of sample points are obtained through iteration, and then the least square method is used to solve the experimental points, thus obtaining the reliability index. The results of calculation examples show that the proposed method can reduce the sampling point range while ensuring the accuracy of calculation, effectively improve the efficiency of the reliability index, and provide a reasonable and effective method for the reliability analysis of the implicit limit state.
\end{abstract}

\section{Introduction}

In the reliability assessment of large and complex structures, the limit state function is often implicit and unknown, which brings great difficulties to the structural reliability analysis. The Monte Carlo method, as a highly accurate method of reliability analysis, has always been favored by researchers, but it is computationally intensive so that it cannot be used in practical engineering [14]. To overcome the problem of computational efficiency, researchers developed a response surface method that approximates the implicit limit state function by a series of deterministic experiments using polynomial functions to reduce the amount of computation within an acceptable error range and improve the computational complexity [5-8].

The early response surface method used linear polynomials or quadratic polynomials to approximate the true limit state function. In Ref.[7], an efficient response surface method that requires only two iterations is proposed by using quadratic polynomials that do not contain crossover terms. Later, Ref. [8] improved it and formed the classical response surface method through multi-step iteration. In the response surface method, the different selection strategies of the experimental points have a great influence on the experimental results. The gradient projection method proposed in Ref. [9] is a method that makes the experimental points fall as far as possible on the equation of limit state. The cumulative response surface law proposed in Ref. [10] makes full use of the information of existing experimental points to select the final experimental point. Because different experimental points have different effects on the solution of implicit limit state equations, there are still some problems in choosing the experimental points. Ref. [11-15] solved the implicit limit state equation by solving the polynomial in a weighted way, which guaranteed a high approximation of the failure probability of the experimental point. Ref. [16] combines weighted thought and nonlinear polynomial to form a weighted nonlinear response surface method and solves the problem of low-dimensional nonlinear state function. Combining the advantages of fractional weighting and exponential weighting with nonlinear polynomials, this paper improves the solving accuracy of weighted nonlinear response surface method in solving nonlinear limit state function. 


\section{Nonlinear Weighted Response Surface Method}

In the process of structural reliability analysis, the most fundamental goal is to find the failure probability $P_{f}$ of the structure by a certain method. The solution formula can be expressed as:

$$
P_{f}=\int_{D} f_{x}(x) d x
$$

Where $x=\left[x_{1}, x_{2}, \cdots, x_{n}\right]^{T}$ is the basic random variable vector, $f_{x}(x)$ is the joint probability density function of the basic random variable, $\mathrm{D}$ is the failure area, which is the area where the limit state function satisfies $g(x) \leq 0$.

When the function is a non-linear function of the basic variables, the mean first order and second moment method linearly expand the function to a Taylor series at the mean point $\mu_{x}=\left(\mu_{x_{1}}, \mu_{x_{2}}, \cdots, \mu_{x_{n}}\right)$ of the basic variable as follows

$$
Z=g\left(x_{1}, x_{2}, \cdots, x_{n}\right) \approx g\left(\mu_{x_{1}}, \mu_{x_{2}}, \cdots, \mu_{x_{n}}\right)+\sum_{i=1}^{n}\left(\frac{\partial g}{\partial x_{i}}\right)\left(x_{i}-\mu_{x_{i}}\right)
$$

Where $\left(\frac{\partial g}{\partial x_{i}}\right)_{\mu_{x}}$ represents the function value of the derivative of the function at the mean point $\mu_{x}$.

According to the linearized function of Eq. (2), the mean $\mu_{g}$ and variance $\sigma_{g}^{2}$ of the function are approximated as follows

$$
\begin{gathered}
\mu_{g}=g\left(\mu_{x_{1}}, \mu_{x_{2}}, \cdots, \mu_{x_{n}}\right) \\
\sigma_{g}^{2}=\sum_{i=1}^{n}\left(\frac{\partial g}{\partial x_{i}}\right)^{2} \sigma_{\mu_{x}}^{2}+\sum_{i=1}^{n} \sum_{j=1, j \neq i}^{n}\left(\frac{\partial g}{\partial x_{i}}\right)_{\mu_{x}}\left(\frac{\partial g}{\partial x_{j}}\right)_{\mu_{x}} \operatorname{Cov}\left(x_{i}, x_{j}\right)
\end{gathered}
$$

If the basic variables are independent of each other, $\sigma_{g}^{2}$ can be simplified as

$$
\sigma_{g}^{2}=\sum_{i=1}^{n}\left(\frac{\partial g}{\partial x_{i}}\right)_{\mu_{x}}^{2} \sigma_{x_{i}}^{2}
$$

In the case of nonlinear function, reliability index $\beta$ and failure probability $P_{f}$ are written as

$$
\beta=\frac{\mu_{g}}{\sigma_{g}}=\frac{g\left(\mu_{x_{1}}, \mu_{x_{2}}, \cdots, \mu_{x_{n}}\right)}{\sqrt{\sum_{i=1}^{n}\left(\frac{\partial g}{\partial x_{i}}\right)^{2} \sigma_{\mu_{x}}^{2}+\sum_{i=1}^{n} \sum_{j=1, j \neq i}^{n}\left(\frac{\partial g}{\partial x_{i}}\right)_{\mu_{x}}\left(\frac{\partial g}{\partial x_{j}}\right)_{\mu_{x}} \operatorname{Cov}\left(x_{i}, x_{j}\right)}}
$$

\subsection{Construction of Weight Matrix}

Suppose the implicit limit state equation $g(x)=0$ in the basic variable $x=\left[x_{1}, x_{2}, \cdots, x_{n}\right]^{T}$ contains n elements, in order to calculate the failure probability $P_{F}$, a quadratic polynomial without cross terms is chosen as the nonlinear response surface function to approximate $g(x)=0$ as shown below

$$
\bar{g}(x)=\mathrm{a}_{0}+\sum_{i=1}^{n} b_{i} x_{i}+\sum_{i=1}^{n} c_{i} x_{i}^{2}
$$

Where $a, b$ and $c$ is $2 n+1$ of undetermined coefficients.

In order to determine the $2 n+1$ undetermined coefficients in the determinant, the $m(m>2 n+1)$ sampling points $x_{j}$ are selected and the real functional function value $g\left(x_{j}\right)$ of the $m$ sampling points are calculated. The undetermined coefficient is usually obtained by the least squares method as follows

$$
B=\left(x^{T} x\right)^{-1} x^{T} y
$$

Where $y$ is the response array corresponding to the experimental point, $x$ is the sampling coefficient matrix with dimensions of $m$ experimental points, which can be expressed as 


$$
a=\left[\begin{array}{ccccccc}
1 & x_{11} & \cdots & x_{1 n} & x_{11}^{2} & \cdots & x_{1 n}^{2} \\
1 & x_{21} & \cdots & x_{2 n} & x_{21}^{2} & \cdots & x_{2 n}^{2} \\
\vdots & \vdots & & \vdots & \vdots & & \vdots \\
1 & x_{l 1} & \cdots & x_{\ln } & x_{l 1}^{2} & \cdots & x_{\ln }^{2}
\end{array}\right]
$$

The weight of the sampling points determines its importance in the regression analysis, indicating that the more important sampling points have the greater weight. As the reliability of the reliability of the improvement depends on the response to the real limit state equation in the design point of the region fitting accuracy, and the real limit state equation can be approximated by a step-by-step approach. Therefore, the distance between the experimental point and the limit state equation $g(x)=0$ can be used to construct the weights.

$\omega_{j}(j=1,2, \cdots, m)$ is taken to represent the weight of each experimental sampling point, and the weight matrix is represented by a $m \times m$-dimensional diagonal matrix $\omega=\operatorname{diag}\left(\omega_{j}\right)$. The undetermined coefficient $b$ can be expressed as follows:

$$
B=\left(x^{T} \omega x\right)^{-1} x^{T} \omega y
$$

The weight matrix $W$ is constructed as follows

$$
\left\{\begin{array}{l}
g_{\text {best }}=\min _{i=1}^{m}\left|g\left(x_{i}\right)\right| \\
\omega_{j}=\exp \left(\frac{g_{\text {best }}}{\left|g\left(x_{i}\right)\right|}\right)
\end{array} \quad j=1,2, \cdots, m\right.
$$

\subsection{Basic Steps}

The proposed nonlinear weighted response surface method can be explained through the following steps.

(1). Choosing a non-linear response surface function approximates an implicit limit state function;

(2). In the first iteration, taking $x_{1}^{*}=\left(x_{11}^{*}, x_{12}^{*}, \cdots, x_{1 n}^{*}\right)$ as the experimental center point, $2 n+1$ sampling points are selected as follows:

$$
\begin{gathered}
x_{1}^{*}=\left(x_{11}^{*}, x_{12}^{*}, \cdots, x_{1 n}^{*}\right) \\
x_{1}^{*}=\left(x_{11}^{*}, \cdots, x_{1 i}^{*}+f \sigma_{x_{i}}, \cdots, x_{1 n}^{*}\right), i=1,2, \cdots, n
\end{gathered}
$$

As the experimental center point $x_{1}^{*}$ is the mean point $u_{x}=\left(u_{x 1}, u_{x 2}, \cdots, u_{x n}\right), \sigma_{x_{i}}$ is the standard deviation of the basic random variable $x_{i}, f$ is the interpolation coefficient, generally taken as a constant between 1 and 3 ;

(3). The real limit state function value corresponding to $2 n+1$ experimental points is calculated by the method of constructing the weights of experimental points and the approximate limit state equation $\bar{g}(x)$ and design point $x_{D}^{(1)}=\left(x_{D 1}^{(1)}, x_{D 2}^{(1)}, \cdots, x_{D n}^{(1)}\right)$ of the response surface of the first iteration are obtained by the least square method;

(4). At the iteration $k(k \geq 2)$, we get the point $g\left(x_{1}^{*(k)}\right)$ close to 0 by the linear interpolation of the remaining point $\left(u_{x}, g\left(u_{x}\right)\right)$ of the design point $\left(x_{D}^{(k-1)}, g\left(x_{D}^{(k-1)}\right)\right)$, and take $x_{1}^{*(\mathrm{k})}$ as the sampling center. The linear interpolation method is as follows:

$$
x_{1 i}^{*(k+1)}=u_{x_{i}}+\left(x_{D i}^{*(k)}-u_{x_{i}}\right) \frac{g\left(u_{x}\right)}{g\left(u_{x_{i}}\right)-g\left(x_{D i}^{*(k)}\right)}
$$

Around $x_{1}^{*(\mathrm{k})}$, a total of $2 n$ new experimental points $\left(x_{11}^{*(\mathrm{k})}, \cdots, x_{1 i}^{*(\mathrm{k})}+f^{(\mathrm{k})} \sigma_{x_{i}}, \cdots, x_{1 n}^{*(\mathrm{k})}\right)$, $(i=1,2, \cdots, n)$ are selected, together with experimental points in the previous $(k-1)$ iterations, which together constitute the experimental point of the $k$-th weighted least-squares regression analysis;

(5). Calculate the weights of $k \times(2 n+1)$ experimental points in the $k$ iteration; 
(6). Take $x_{i}=\left(x_{i 1}, x_{i 2}, \cdots, x_{i n}\right)(i=1,2, \cdots, l)$ as $l=k \times(2 n+1)$ experimental points, the regression matrix composed of $l$ experimental points is denoted as $\hat{x}$, and the weighted least squares method is used to determine the undetermined coefficient vector $B$ of the second polynomial $k$ iteration without cross terms;

(7). According to the method of reliability analysis of explicit limit state function, the design point $x_{D}^{(k)}$ and the reliability index $\beta^{(k)}$ of the response surface equation $\bar{g}^{(k)}=0$ of the $k$-th iteration are obtained;

(8). Determine whether the relative error of the reliability index obtained by the two iterations meets the requirement. According to $\left|\frac{\beta^{(k)}-\beta^{(k-1)}}{\beta^{(k-1)}}\right|<\xi$ ( $\xi$ is the set of error criteria) to determine the reliability index is satisfied, if satisfied then go to step (9), otherwise go to step (4);

(9). The failure probability calculated by the output response surface method. The failure probability can be obtained by the Monte Carlo method or calculated by the first order and second moment method.

\section{Numerical Examples}

In order to verify the rationality and feasibility of proposed method, the following examples are used to calculate the failure probability of the implicit limit state equation. Different calculation methods are used to calculate the examples and the results are compared.

Example 1

An exponential limit state function is considered:

$g(x)=\exp \left[0.4\left(x_{1}+2\right)+6.4\right]-\exp \left[0.3 x_{2}+5.0\right]-200$

Where $x_{i} \sim N(0,1)(i=1,2)$. The results of different calculation methods are reported in Table 1.

Table 1 Compared results - Example 1

\begin{tabular}{ccccc}
\hline Reference/Method & Sample size & $\beta$ & $P_{F}$ & Error(\%) \\
\hline Monte Carlo & 106 & 2.689 & $3.58 \times 10^{-3}$ & 0 \\
RSM & 85 & 1.945 & $2.584 \times 10^{-2}$ & -2.766 \\
proposed method & 68 & 2.987 & $2.338 \times 10^{-3}$ & 1.108 \\
\hline
\end{tabular}

It can be seen from the results that using the weighted response surface method for the exponential equation can calculate the reliability index and failure probability with smaller error, which is greatly improved compared with the classical response surface method. It shows that the weighted response surface method can improve the efficiency of exponential example calculations.

Example 2.

A cubic limit state function is considered:

$g(x)=x_{1}^{3}+x_{1}^{2} x_{2}+x_{2}^{3}-18$

Where $x_{i} \sim N(0,1)(i=1,2)$. The results of different calculation methods are reported in Table 2 .

Table 2. Compared results - Example 2

\begin{tabular}{ccccc}
\hline Reference/Method & Sample size & $\beta$ & $P_{F}$ & Error(\%) \\
\hline Monte Carlo & 106 & 2.533 & $5.63 \times 10^{-3}$ & 0 \\
RSM & 168 & 1.945 & $2.652 \times 10^{-2}$ & -2.321 \\
proposed method & 147 & 2.201 & $1.383 \times 10^{-2}$ & -1.310 \\
\hline
\end{tabular}

It can be seen from the results that the weighted response surface method can calculate the reliability index and the failure probability with smaller error for the exponential type equation, which has a higher accuracy than the classical response surface method. The computational efficiency of the limit state function has also increased considerably. 


\section{Example 3}

Limit state function with derivative term is considered, the performance function is given as

$$
g(x)=x_{1}-\frac{x_{2}}{x_{3}}
$$

Where $x_{1} \sim N(600.0,30.0), x_{2} \sim N(1000.0,33.0), x_{3} \sim N(2.0,0.1)$.The comparison of the results of different calculation methods are reported in Table 3.

Table 3. Compared results — Example 3

\begin{tabular}{ccccc}
\hline Reference/Method & Sample size & $\beta$ & $P_{F}$ & Error(\%) \\
\hline Monte Carlo & 105 & 1.283 & $1.21 \times 10^{-2}$ & 0 \\
RSM & 35 & 1.136 & $1.13 \times 10^{-2}$ & -1.145 \\
proposed method & 36 & 1.249 & $1.23 \times 10^{-2}$ & -0.265 \\
\hline
\end{tabular}

The result of the example analysis shows that the method of nonlinear weighted response surface method has higher accuracy. In the process of solving the same number of samples, the calculation accuracy is better than the traditional response surface method, and the response time is fast. The results obtained by using the standard algorithm have high precision but consume a large amount of solving time, and the computational efficiency becomes low resulting in the practical application constrained.

\section{Summary}

In this paper, an improved weighted nonlinear response surface method is used to solve the reliability problem of implicit limit state equations. By combining the fractional weighting method with the exponential weighting method, the experimental point can be fitted to the implicit limit state equation to the maximum extent to improve the computational efficiency. Through the example analysis, it can be seen that the method in this paper can improve the accuracy of calculation with a little practical increase.

\section{Acknowledgements}

National Natural Science Foundation of China (No.20130101059JC)

\section{References}

[1] Hasofer AM, Lind NC. An exact and invariant first order reliability format [J]. Journal of Engineering Mechanics, ASCE, 1974, 100(1): 111-121.

[2] Song Yunlian, Li Shujun, Liu Wenbai. Farther precision of the AFOSM for analyzing the structural reliability [J]. Chinese Journal of Computational Mechanics, 2003, 20(1): 121-126.

[3] Bezerra M A, Santelli R E, Oliveira E P, et al. Response surface methodology (RSM) as a tool for optimization in analytical chemistry[J]. Talanta, 2008, 76(5):965.

[4] Turkstra C, Grigoriu M. Generalized second-moment reliability analysis [J]. American Society of Mechanical Engineers, 1978, 7:1-8.

[5] Lin K, Qiu H, Gao L, et al. Comparison of Stochastic Response Surface Method and Response Surface Method for Structure Reliability Analysis[C]. International Conference on Intelligent Computation Technology and Automation. IEEE, 2009:172-175.

[6] Su C, Li P F, Han D J. Neumann-expansion response surface method for calculating structure reliability[J]. Journal of South China University of Technology, 2009, 37(9):13-12.

[7] Zhao W, Fan F, Wang W. Non-linear partial least squares response surface method for structural reliability analysis[J]. Reliability Engineering \& System Safety, 2017, 161:69-77. 
[8] Rajashekhar MR, Ellingwood BR. A new look at the response safe approach for reliability analysis [J]. Structural Safety, 1993, 12(3):205-220.

[9] Fang Y, Chen J. An improved genetic algorithm for structural reliability response surface method[J]. Chinese Journal of Applied Mechanics, 2017.

[10]Das P K, Zheng Y. Cumulative formation of response surface and its use in reliability analysis. Probabilistic Engineering Mechanics, 2000, 15(4): 309-315.

[11]Kaymaz I, McMahon C A. A response surface method based on weighted regression for structural reliability analysis. Probabilistic Engineering Mechanics, 2005, 20(1):11-17.

[12] Wong S M, Hobbs R E, Onof C. An adaptive response surface method for reliability analysis of structures with multiple loading sequences. Structural Safety, 2005, 27(4):287-308.

[13]Kong X G, Liu J Q, Cao C Q. An Improved Response Surface Method for Reliability Analysis of Plate on Elastic Foundation[J]. Applied Mechanics \& Materials, 2013, 394(394):349-352.

[14]LU Zhen-zhou, YANG Zi-zheng, ZHAO Jie. An Artificial Neural Network Method for Reliability An alysis Basedon Weighted Linear Response Surface. Acta Aeronautica ET Astronautica Sinica,2006,27(6): 1063-1067.

[15]Basaga H B, Bayraktar A, Kaymaz I. An improved response surface method for reliability analysis of structures[J]. Structural Engineering \& Mechanics, 2012, 42(2):175-189.

[16] Gavin H P, Yau S Y. High-order limit state function in the response surface method for structural reliability analysis. Structural Safety, 2008, 30(2): 162-179. 